\title{
Recurrent Inhibitory Network among Striatal Cholinergic Interneurons
}

\author{
Matthew A. Sullivan, Huanmian Chen, and Hitoshi Morikawa \\ Waggoner Center for Alcohol and Addiction Research, Section of Neurobiology and Institute for Cellular and Molecular Biology, The University of Texas at \\ Austin, Austin, Texas 78712
}

The striatum plays a central role in sensorimotor learning and action selection. Tonically active cholinergic interneurons in the striatum give rise to dense axonal arborizations and significantly shape striatal output. However, it is not clear how the activity of these neurons is regulated within the striatal microcircuitry. In this study, using rat brain slices, we find that stimulation of intrastriatal cholinergic fibers

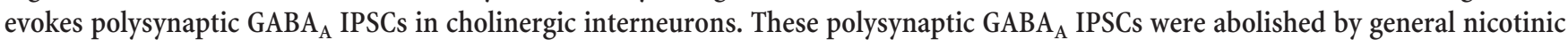
acetylcholine receptor antagonists and also by a specific antagonist of nicotinic receptors containing $\beta 2$ subunits. Dopamine receptor antagonists or dopamine depletion failed to block polysynaptic IPSCs, indicating that phasic dopamine release does not directly mediate the polysynaptic transmission. Dual recording from pairs of cholinergic interneurons revealed that activation of a single cholinergic

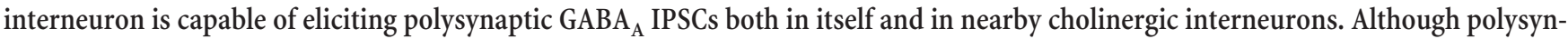
aptic transmission arising from a single cholinergic interneuron was depressed during repetitive $2 \mathrm{~Hz}$ firing, intrastriatal stimulation reliably evoked large polysynaptic IPSCs by recruiting many cholinergic fibers. We also show that polysynaptic GABAergic inhibition leads to a transient suppression of tonic cholinergic interneuron firing. We propose a novel microcircuit in the striatum, in which cholinergic interneurons are connected to one another through GABAergic interneurons. This may provide a mechanism to convert activation of cholinergic interneurons into widespread recurrent inhibition of these neurons via nicotinic excitation of striatal GABAergic neurons.

Key words: striatum; microcircuit; cholinergic neuron; acetylcholine; ACh; nicotinic receptor; patch clamp

\section{Introduction}

The striatum, the major input structure of the basal ganglia, plays a pivotal role in sensorimotor learning and action selection. It receives glutamatergic input from the cortex and thalamus and dopaminergic input from the midbrain, and then conveys the integrated information to downstream basal ganglia nuclei (Graybiel et al., 1994). GABAergic spiny projection neurons, which comprise the vast majority ( $>95 \%$ in rodents) of striatal neurons, provide the sole output of the striatum. Accumulating evidence indicates an important role of the local microcircuitry in regulating the activity of spiny projection neurons. Although spiny neurons form a weak lateral inhibitory network among themselves via local axon collaterals, feedforward inhibition by different types of GABAergic interneurons exerts more powerful control of spiny neuron excitability (Tepper and Bolam, 2004; Tepper et al., 2004).

The striatum contains another class of neurons, i.e., the cholinergic interneurons. These neurons are thought to correspond

\footnotetext{
Received May 15, 2008; revised July 9, 2008; accepted July 21, 2008.

This work was supported by National Institutes of Health Grant R01DA015687. We thank Dr. Charles Wilson for comments on this manuscript. We also thank Clifford Buckley for conducting some preliminary experiments.

Correspondence should be addressed to Hitoshi Morikawa, Section of Neurobiology, The University of Texas at

Austin, 2415 Speedway, PAT 402, Austin, TX 78712. E-mail: morikawa@mail.utexas.edu.

H. Chen's present address: Department of Neuroscience, Johns Hopkins University, Baltimore, MD 21205.

DOI:10.1523/JNEUROSCI.2411-08.2008

Copyright $\odot 2008$ Society for Neuroscience $\quad 0270-6474 / 08 / 288682-09 \$ 15.00 / 0$
}

to the tonically active neurons (TANs) recorded in vivo, which respond to sensory stimuli with a transient depression, or pause, of tonic firing during sensorimotor conditioning (Wilson et al., 1990; Aosaki et al., 1994a; Reynolds et al., 2004; Apicella, 2007). They give rise to extensive axonal arbors forming a dense plexus of cholinergic fibers within the striatum. Although cholinergic interneurons constitute merely $1-2 \%$ of the striatal neuronal population, they can significantly impact striatal output and functions because of their tonic activity and dense local innervation (Zhou et al., 2002; Kitabatake et al., 2003). Indeed, numerous cholinergic responses have been reported that are caused by different types of nicotinic and muscarinic acetylcholine (ACh) receptors on presynaptic and postsynaptic elements in the striatum (Calabresi et al., 2000; Zhou et al., 2003). In particular, transient reduction in ACh release during the pause of tonic cholinergic neuron activity has been implicated in the control of glutamatergic and GABAergic transmission onto spiny projection neurons (Wang et al., 2006; Narushima et al., 2007; Pakhotin and Bracci, 2007).

A variety of intrinsic conductances enable cholinergic interneurons to fire autonomously (Bennett et al., 2000; Wilson, 2005). Thus, their firing pattern is determined by the dynamic interplay between this powerful intrinsic mechanism and the synaptic inputs they receive. For example, glutamatergic afferents from the cortex and thalamus have been implicated in triggering and/or synchronizing the pause response of these neurons (Ma- 
A
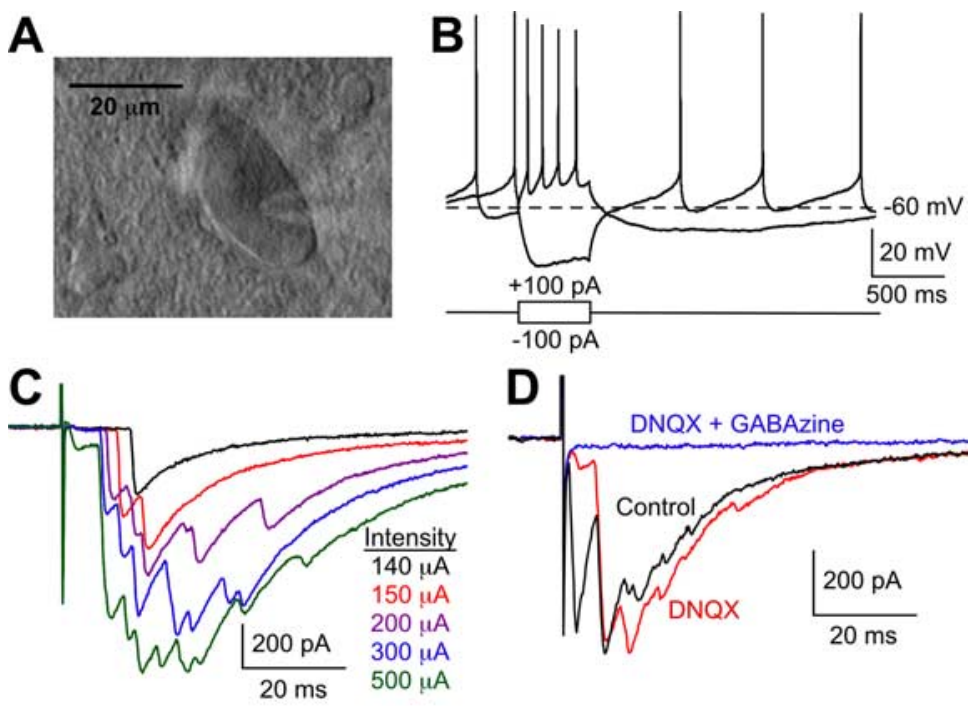

E

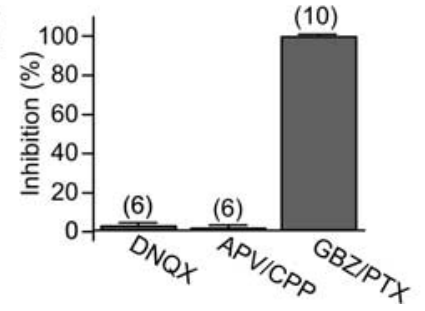

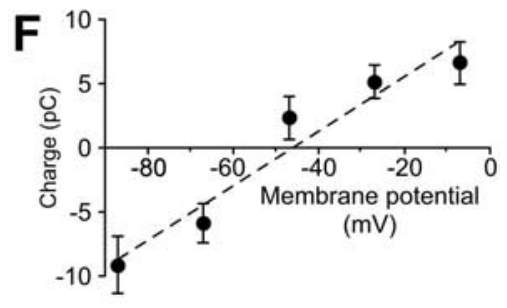

Figure 1. Intrastriatal stimulation elicits monosynaptic and polysynaptic GABA $\mathrm{IPSC}$ in cholinergic interneurons. $\boldsymbol{A}$, Photomicrograph of a recorded cholinergic interneuron in a striatal slice. $\boldsymbol{B}$, Representative traces of a spontaneously firing cholinergic interneuron depicting its response to positive and negative current injections. $C$, A series of PSC traces evoked by intrastriatal stimulation of various intensities. The experiment was done in the presence of DNQX $(10 \mu \mathrm{M}) \cdot \boldsymbol{D}$, Representative traces of PSCs in control (black), in DNQX (red), and in DNQX + GABAzine (10 $\mu$ m; blue). $E$, Summary bar graph showing the effects of DNQX (10 $\mu \mathrm{M}), \operatorname{APV}(100 \mu \mathrm{M})$, or (PP $(100 \mu \mathrm{M})$, and GABAzine $(\mathrm{GBZ} ; 10 \mu \mathrm{M})$ or picrotoxin (PTX; $100 \mu \mathrm{M})$ on the late polysynaptic component of PSCS. F, Summary graph plotting the charge transfer of the polysynaptic component versus the holding potential $(n=12)$. The dotted line represents linear fit to the data.

tsumoto et al., 2001; Reynolds and Wickens, 2004; Reynolds et al., 2004). However, it is not well understood how the activity of cholinergic interneurons is regulated by local GABAergic and cholinergic networks in the striatum.

In this study, we show that activation of intrastriatal cholinergic fibers elicits polysynaptic $\mathrm{GABA}_{\mathrm{A}}$ IPSCs in cholinergic interneurons recorded in brain slices. Excitation of striatal GABAergic neurons via nicotinic acetylcholine receptors (nAChRs) mediates this polysynaptic inhibition in a manner independent of dopamine. Intriguingly, we have further found that activation of a single cholinergic interneuron is capable of eliciting polysynaptic $\mathrm{GABA}_{\mathrm{A}}$ IPSCs onto itself and nearby cholinergic interneurons. These findings provide an important insight into the striatal microcircuitry controlling cholinergic neuron excitability.

\section{Materials and Methods}

Slice preparation and recordings. All animal procedures were in accordance with the National Institutes of Health guidelines and approved by the Institutional Animal Care and Use Committee at The University of Texas, Austin. Oblique horizontal slices $\left(30-45^{\circ}, 230 \mu \mathrm{m}\right)$ containing the dorsal striatum were prepared from male Sprague Dawley rats (21-28 d). Slices were cut using a Vibratome (VT1000S; Leica) in ice-cold saline containing (in mM) $2.5 \mathrm{KCl}, 1.25 \mathrm{NaH}_{2} \mathrm{PO}_{4}, 7.5 \mathrm{MgCl}_{2}, 0.5 \mathrm{CaCl}_{2}, 10$ glucose, 205 sucrose, $25 \mathrm{NaHCO}_{3}$ (saturated with $95 \% \mathrm{O}_{2}$ and $5 \% \mathrm{CO}_{2}$ ), and then incubated at $35^{\circ} \mathrm{C}$ for $>1 \mathrm{~h}$ in physiological saline containing (in mM) $126 \mathrm{NaCl}, 2.5 \mathrm{KCl}, 1.2 \mathrm{NaH}_{2} \mathrm{PO}_{4}, 1.2 \mathrm{MgCl}_{2}, 2.4 \mathrm{CaCl}_{2}, 11$ glucose, $21.4 \mathrm{NaHCO}_{3}, 5$ kynurenic acid (saturated with $95 \% \mathrm{O}_{2}$ and $5 \%$

$\mathrm{CO}_{2}, \sim 295 \mathrm{mOsm} / \mathrm{kg}$ ). Recordings were made at $35^{\circ} \mathrm{C}$ in the same saline (without kynurenic acid) perfused at $2-3 \mathrm{ml} / \mathrm{min}$.

Cells were visualized using an upright microscope (BX51WI; Olympus) with infrared/differential interference contrast or oblique illumination optics. Whole-cell recordings were made with borosilicate glass pipettes (1.6-2.2 $\mathrm{M} \Omega$ ) filled with internal solution containing the following (in mM): $115 \mathrm{~K}$-methylsulfate, 20 $\mathrm{KCl}, 1.5 \mathrm{MgCl}_{2}$, 10 HEPES, 0.025 EGTA, $2 \mathrm{Mg}$ ATP, $0.2 \mathrm{Na}_{2}-\mathrm{GTP}$, and $10 \mathrm{Na}_{2}$ phosphocreatine $(\mathrm{pH} 7.25-7.3, \sim 280 \mathrm{mOsm} / \mathrm{kg})$. Data were acquired using a Multiclamp 700A or 700B amplifier (Molecular Devices), filtered at $2-5 \mathrm{kHz}$, digitized at $5-20 \mathrm{kHz}$, and collected using Axograph X softwear (Axograph Scientific). The membrane potential was corrected for a liquid junction potential of $7 \mathrm{mV}$.

Dopamine depletion. Rats were injected intraperitoneally with the vesicular monoamine transporter inhibitor reserpine $(5 \mathrm{mg} / \mathrm{kg}, 24 \mathrm{~h}$ before dissection) and the tyrosine hydroxylase inhibitor $\alpha$-methyl-para-tyrosine (AMPT) (300 mg/kg, $4 \mathrm{~h}$ before dissection, plus $200 \mathrm{mg} /$ $\mathrm{kg}, 2 \mathrm{~h}$ before dissection). Slices were preincubated in reserpine $(1 \mu \mathrm{M})$, AMPT $(30 \mu \mathrm{M})$, and D-amphetamine $(1 \mu \mathrm{M})$, and recordings were done in the continuous presence of reserpine (1 $\mu \mathrm{M})$ and AMPT $(30 \mu \mathrm{M})$ to ensure complete depletion of dopamine.

Drugs. 6,7-Dinitroquinoxaline-2,3-dione (DNQX), 4-hydroxyquinoline-2-carboxylic acid (kynurenic acid), D-(-)-2-amino-5phosphonopentanoic acid (APV), 3- ( $R)-2-$ carboxypiperazin-4-yl)-propyl-1-phosphonic acid (CPP), (5R,10S)-(-)-methyl-10,11dihydro-5H-dibenzo $[a, d]$ cyclohepten-5-imine maleate (MK-801), dihydro- $\beta$-erythroidine hydrobromide $(\mathrm{DH} \beta \mathrm{E})$, and $(R)-(+)-7$ chloro-8-hydroxy-3-methyl-1-phenyl-2,3,4,5-tetrahydro- $1 H$-3-benzazepine hydrochloride (SCH23390) were obtained from Tocris Bioscience. All other chemicals were obtained from Sigma.

Data analysis. Data are expressed as means \pm SEM. Statistical significance was determined with Student's $t$ test. The difference was considered significant at $p<0.05$. The onset of suppression in tonic firing was defined as the first of three consecutive bins (bin width, $20 \mathrm{~ms}$ ) that deviated significantly from the baseline firing frequency before the stimulus, whereas the offset was defined as the first of three consecutive bins that returned to the baseline level (Aosaki et al., 1995).

\section{Results}

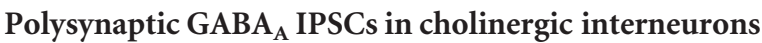

Cholinergic interneurons were identified by their large soma (25-50 $\mu \mathrm{m})$ and unique electrophysiological properties (Fig. $1 A, B)$, such as spontaneous action potential firing, a longduration afterhyperpolarization, and a depolarizing sag in response to hyperpolarizing current injection (Wilson, 2005). To investigate local synaptic inputs onto these neurons, we made whole-cell voltage-clamp recordings at a holding potential of $-87 \mathrm{mV}$ and measured synaptic currents evoked every $20 \mathrm{~s}$ by intrastriatal stimulation using a bipolar electrode (100-200 $\mu \mathrm{m}$ tip separation) placed $100-300 \mu \mathrm{m}$ from the recorded cell. This causes stimulation of both intrinsic fibers from striatal neurons and extrinsic inputs from other structures. Recordings were done in the presence of an AMPA/kainate receptor antagonist, DNQX $(10 \mu \mathrm{M})$, to eliminate responses produced by stimulation of glu- 
tamatergic fibers from the cortex and thalamus (Lapper and Bolam, 1992). A single stimulus induced compound PSCs containing multiple peaks in seven cells tested with various stimulus intensities $(50-800$ $\mu \mathrm{A}, 0.2 \mathrm{~ms}$ ) (Fig. 1C). At threshold intensity $(50-150 \mu \mathrm{A})$, a single peak $(150 \pm 29$ pA) was elicited with a long latency $(14.2 \pm 0.6 \mathrm{~ms})$ after the stimulus. The PSC increased in amplitude, duration, and number of peaks with an increase in stimulus intensity. At the same time, the onset latency became shorter up to a certain level in each cell, ranging from 6.6 to $10.8 \mathrm{~ms}$ $(8.2 \pm 0.7 \mathrm{~ms})$, consistent with the idea that it is evoked polysynaptically. Higherintensity stimulation produced an additional component (30-200 pA) that occurred with a much shorter latency (1.2$3.6 \mathrm{~ms}, 2.1 \pm 0.3 \mathrm{~ms})$. The latency to this early component was not changed by further increase in stimulus intensity, implying that it is a monosynaptic response. The range of the onset latency of the two components had no overlap. Furthermore, the late component was selectively suppressed with an increase in extracellular concentrations of divalent cations $\left(4 \mathrm{mM} \mathrm{Ca}^{2+}\right.$ and $4 \mathrm{mM} \mathrm{Mg}^{2+}, n=4$ ) (supplemental Fig. S1, available at www.jneurosci.org as supplemental material), a treatment commonly used to block polysynaptic responses by elevating action potential threshold (Cruikshank et al., 2002; Liao and Walters, 2002). Therefore, the early and late components represent monosynaptic and polysynaptic PSCs, respectively.

GABAergic transmission makes a major contribution to the striatal microcircuitry (Tepper and Bolam, 2004; Tepper et al., 2004). Indeed, both monosynaptic and polysynaptic PSCs were completely blocked by bath application of $\mathrm{GABA}_{\mathrm{A}}$ receptor antagonists, GABAzine $(10 \mu \mathrm{M})$ or picrotoxin $(100 \mu \mathrm{M})$ (Fig. $1 D, E)$. Furthermore, the charge transfer mediated by the polysynaptic component reversed at $-45 \pm 3 \mathrm{mV}(n=12)$ (Fig. $1 F)$, close to the estimated equilibrium potential for $\mathrm{Cl}^{-}\left(E_{\mathrm{Cl}}=\right.$ $-47 \mathrm{mV}$ ) under our recording conditions, as would be expected for $\mathrm{Cl}^{-}$-permeable $\mathrm{GABA}_{\mathrm{A}}$ conductance. Together, these results demonstrate that intrastriatal stimulation produces rapid monosynaptic and delayed polysynaptic $\mathrm{GABA}_{\mathrm{A}}$ IPSCs in cholinergic interneurons under AMPA/kainate receptor blockade. It should

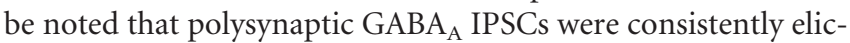
ited in all cholinergic interneurons tested for intrastriatal stimulation in this study. In contrast, only monosynaptic IPSCs, without any polysynaptic IPSCs, were observed in spiny projection neurons ( $n=8$, data not shown). These monosynaptic IPSCs in spiny neurons and cholinergic interneurons are most likely caused by stimulation of intrinsic GABAergic fibers from striatal GABAergic neurons, although extrinsic GABAergic inputs from the globus pallidus may also be involved (Kita, 2007).

It has been shown that electrical stimulation of the subcortical white matter, which contains corticostriatal and thalamostriatal glutamatergic fibers, produces polysynaptic GABA $_{\mathrm{A}}$ IPSCs in cholinergic interneurons via AMPA receptor-dependent excitation of striatal GABAergic neurons (Suzuki et al., 2001). To test whether the intrastriatal stimulation described above can invoke

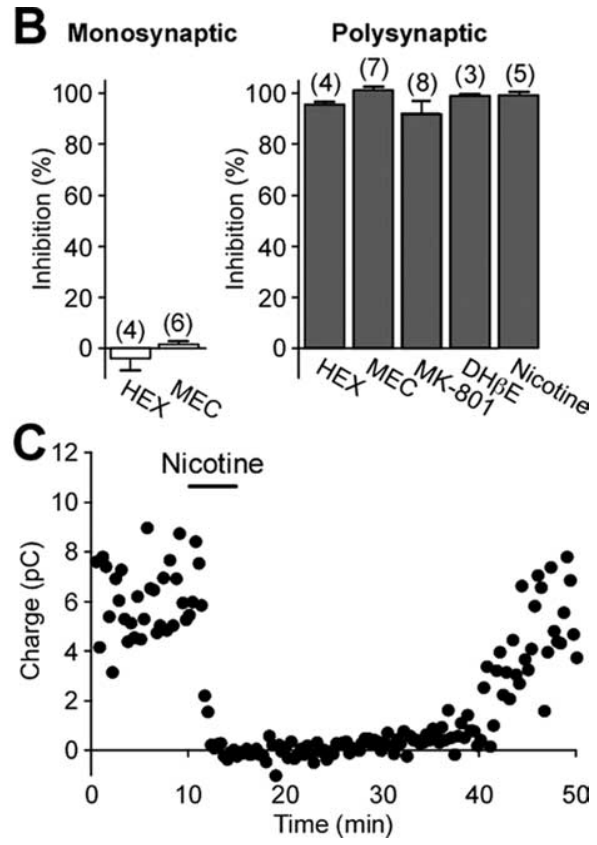

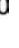

Figure 2. $\beta 2$-Containing $n A C h R s$ mediate polysynaptic $\mathrm{GABA}_{\mathrm{A}} \mathrm{IPSC}$. $\boldsymbol{A}$, Representative traces of $\mathrm{GABA}_{\mathrm{A}}$ IPSC illustrating the nents of IPSCS. C, Time graph of a representative experiment depicting the effect of nicotine ( $500 \mathrm{~nm})$ on the charge carried by polysynaptic GABA $\mathrm{APSC}$. Note the slow time course of recovery.

this mechanism, we also recorded PSCs in the absence of DNQX. Intrastriatal stimulation $(200-800 \mu \mathrm{A})$ produced a large monosynaptic component $(50-600 \mathrm{pA})$ with a short latency $(2.6 \pm 0.1$ $\mathrm{ms}, n=10)$ followed by a delayed polysynaptic component. The early component was mostly reduced by DNQX $(10 \mu \mathrm{M})$ and abolished by the combination of DNQX and GABAzine $(10 \mu \mathrm{M}$; $n=4)$ or picrotoxin $(100 \mu \mathrm{M} ; n=4)$ (Fig. $1 D)$, indicating that it represents a mixture of monosynaptic AMPA EPSCs and $\mathrm{GABA}_{\mathrm{A}}$ IPSCs. In contrast, DNQX had no effect on the late component, which was abolished by subsequent application of GABAzine or picrotoxin, as described above (Fig. $1 D, E$ ). Furthermore, NMDA receptor antagonists APV $(100 \mu \mathrm{M})$ and $\mathrm{CPP}(100 \mu \mathrm{M})$ failed to affect the late component (Fig. $1 E$ ). Therefore, activation of glutamatergic fibers does not contribute to the generation of polysynaptic $\mathrm{GABA}_{\mathrm{A}}$ IPSCs by intrastriatal stimulation. All of the experiments described hereafter were performed in the presence of DNQX to eliminate monosynaptic AMPA EPSCs.

\section{$\boldsymbol{\beta} 2$-Containing $\mathrm{nAChRs}$ mediate polysynaptic GABA $\mathrm{A}_{\mathrm{A}}$ IPS}

It is possible that polysynaptic $\mathrm{GABA}_{\mathrm{A}}$ IPSCs are mediated by stimulation of local cholinergic fibers, causing excitation of striatal GABAergic neurons via nAChRs. To test this possibility, we examined the effects of different $\mathrm{nAChR}$ antagonists. Bath application of general $\mathrm{nAChR}$ antagonists, hexamethonium (10-50 $\mu \mathrm{M})$ and mecamylamine $(1-10 \mu \mathrm{M})$, selectively abolished the polysynaptic component of $\mathrm{GABA}_{\mathrm{A}}$ IPSCs without affecting the early monosynaptic component (Fig. 2A,B). MK-801, a commonly used NMDA receptor antagonist, is known to potently block nAChRs, as well (Amador and Dani, 1991; Yamakura et al., 2000). Indeed, MK-801 (20-100 $\mu \mathrm{M})$ also inhibited the polysynaptic component by $92 \pm 5 \%(n=8)$ (Fig. $2 B)$. Furthermore, $\mathrm{DH} \beta \mathrm{E}(100 \mathrm{nM})$, a selective antagonist of nAChRs containing $\beta 2$ subunits, completely suppressed the polysynaptic component 

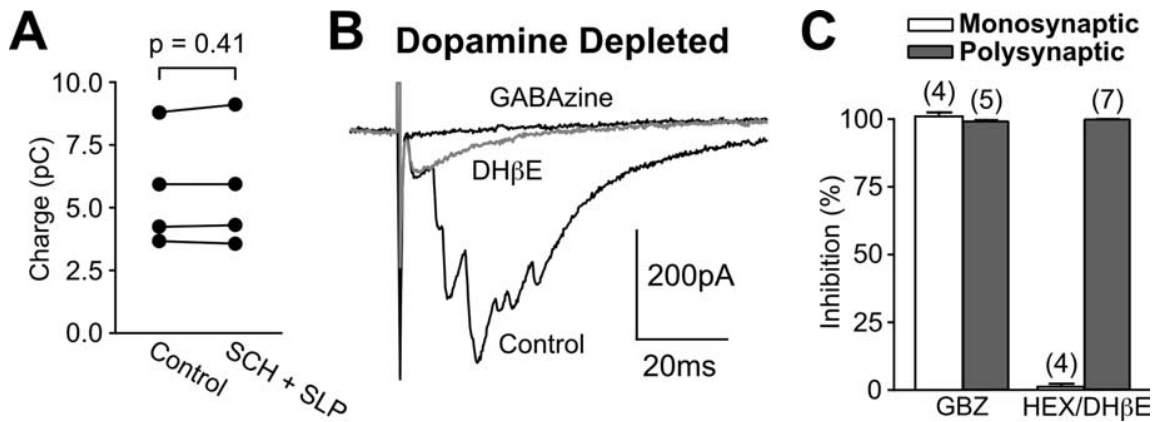

Figure 3. Dopamine does not mediate polysynaptic IPSCs. $\boldsymbol{A}$, The charge carried by polysynaptic IPSC before and after coapplication (7-10 min) of SCH 23390 (SCH; $1 \mu \mathrm{M}$ ) and sulpiride (SLP; $10 \mu \mathrm{M})$ is plotted for individual cells $(n=4)$. $\boldsymbol{B}$, An example trace of an IPSC having both monosynaptic and polysynaptic components in a cholinergic interneuron from a dopamine-depleted rat. A trace (gray) in $\mathrm{DH} \beta \mathrm{E}$ (100 nM) and that in GABAzine (10 $\mu \mathrm{m})$ are also shown. C, Summary bar graph illustrating the effects of GABAzine (GBZ; $10 \mu \mathrm{m}$ ) and hexamethonium (HEX; $50 \mu \mathrm{m}$ ) or DH $\beta E$ (100 nM) on monosynaptic and polysynaptic components of IPSCs in cholinergic interneurons from dopamine-depleted rats.

(Fig. $2 A, B)$. $\beta 2$-Containing $\mathrm{nAChRs}$ are readily desensitized by relatively low concentrations of nicotine $(<1 \mu \mathrm{M})$ (Giniatullin et al., 2005). Consistent with the involvement of $\beta 2$-containing

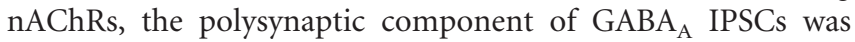
eliminated by bath application of nicotine $(500 \mathrm{nM})$ for $\sim 5 \mathrm{~min}$ (Fig. 2A-C). Recovery from this desensitizing effect of nicotine required tens of minutes, in agreement with the recovery time course of the effects of nicotine on $\beta 2$-containing nAChRmediated responses in previous studies using brain slices (Zhou et al., 2001; Mansvelder et al., 2002). During this slow recovery, the number of peaks in IPSCs gradually increased (Fig. $2 \mathrm{~A}$, bottom trace), suggesting a gradual increase in the number of spikes in GABAergic neurons mediating polysynaptic transmission. Scopolamine $(1 \mu \mathrm{M})$, a muscarinic ACh receptor antagonist, had

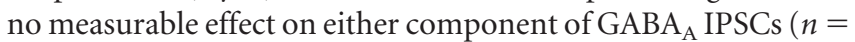
3 , data not shown). These results demonstrate that stimulation of intrastriatal cholinergic fibers excites GABAergic neurons via activation of $\beta 2$-containing $\mathrm{nAChRs,} \mathrm{inducing} \mathrm{polysynaptic}$ $\mathrm{GABA}_{\mathrm{A}}$ IPSCs in cholinergic interneurons.

Recent evidence indicates that phasic activation of $\beta 2$ containing $\mathrm{nAChRs}$ expressed on dopaminergic terminals facilitates dopamine release in the striatum (Zhou et al., 2001; Rice and Cragg, 2004; Zhang and Sulzer, 2004). To examine the involvement of dopamine in the polysynaptic transmission, we next tested dopamine receptor antagonists. Combined application of a $\mathrm{D}_{1}$-like receptor antagonist, $\mathrm{SCH} 23390(1 \mu \mathrm{M})$, and a $\mathrm{D}_{2}$-like receptor antagonist, sulpiride $(10 \mu \mathrm{M})$, had no significant effect on polysynaptic IPSCs $(n=4)$ (Fig. $3 A$ ). Moreover, polysynaptic IPSCs were easily evoked in slices prepared from dopaminedepleted rats $(n=8)$ (Fig. $3 B)$. We further confirmed that these polysynaptic IPSCs evoked in dopamine-depleted slices were blocked by GABAzine (10 $\mu \mathrm{M}, n=5)$, hexamethonium (50 $\mu \mathrm{M}$, $n=5)$, or $\mathrm{DH} \beta \mathrm{E}(100 \mathrm{~nm}, n=2)$ (Fig. $3 B, C)$. From these results, we conclude that dopamine is not directly involved in the generation of nAChR-mediated polysynaptic $\mathrm{GABA}_{\mathrm{A}}$ IPSCs.

\section{Activation of a single cholinergic interneuron elicits polysynaptic $\mathrm{GABA}_{\mathrm{A}}$ IPSCs}

We next asked whether activation of a single cholinergic interneuron can drive polysynaptic inhibition. To test this, an unclamped action potential was evoked every $20 \mathrm{~s}$ by a $2 \mathrm{~ms}$ depolarizing pulse in cholinergic interneurons voltage-clamped at $-87 \mathrm{mV}$. These experiments were done in the presence of a muscarinic ACh receptor antagonist, scopolamine $(1 \mu \mathrm{M})$. The gen- eration of an action potential was confirmed by a large action current during the voltage step (Cui et al., 2007). This resulted in a "feedback" PSC in the same neuron in 9 of 428 neurons tested $(197 \pm 35 \mathrm{pA}$, $16.0 \pm 1.2 \mathrm{~ms}$ latency from the peak of action current) (Fig. 4). We further performed dual recordings from pairs of cholinergic interneurons (20-150 $\mu \mathrm{m}$ apart). An unclamped action potential evoked in one neuron produced a "feedforward" PSC in the other neuron in 7 of 199 pairs $(136 \pm 50 \mathrm{pA}, 13.8 \pm 1.1 \mathrm{~ms}$ latency). The mean success rate to cause a PSC with each action potential was $48 \pm 5 \%$ in these 16 cases. Interestingly, both feedback and feedforward PSCs were observed in three pairs. In two of these three pairs, activation of one of the two neurons evoked PSCs in itself and in the other neuron (an example is illustrated in Fig. $4 A-C$ ), whereas in the other pair, PSCs were induced in only one of the two neurons by activation of either neuron. In the former case, the induction of feedback PSCs and that of feedforward PSCs succeeded or failed together with each action potential, suggesting that both neurons in the pair were contacted by the same GABAergic neuron(s) (Fig. 4B,C). Reciprocal feedforward PSCs were not observed in 199 pairs tested. These PSCs elicited by an action potential in a single presynaptic neuron routinely had only one peak, although two to three peaks were occasionally detected in 2 of the 16 cases (Fig. $4 D$ ), suggesting multiple spikes in a GABAergic neuron connecting two cholinergic interneurons in the pair and/or recruitment of multiple GABAergic neurons.

Feedback $(n=5)$ and feedforward $(n=5)$ PSCs were completely abolished by both $\mathrm{DH} \beta \mathrm{E}(100 \mathrm{nM})$ and GABAzine (10 $\mu \mathrm{M}$ ) (Fig. $4 B, E$ ), consistent with the idea that they are polysynaptic GABA $_{\mathrm{A}}$ IPSCs mediated by activation of GABAergic neurons via $\beta 2$-containing $\mathrm{nAChRs.} \mathrm{In} \mathrm{line} \mathrm{with} \mathrm{this,} \mathrm{PSCs} \mathrm{reversed}$ at $-49 \pm 3 \mathrm{mV}(n=3)$, close to the estimated $E_{\mathrm{Cl}}$ of $-47 \mathrm{mV}$ (Fig. $4 F$ ). These results show that action potential firing in a single cholinergic interneuron can induce polysynaptic IPSCs in both itself and nearby cholinergic interneurons.

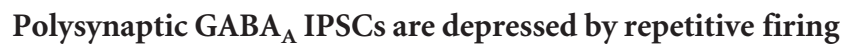
Cholinergic interneurons fire tonically at $\sim 1-8 \mathrm{~Hz}$ both in vitro and in vivo (Wilson et al., 1990; Bennett et al., 2000). If action potentials in single cholinergic interneurons are capable of eliciting polysynaptic IPSCs, one would expect to observe many spontaneous polysynaptic IPSCs. However, we rarely detected large spontaneous IPSCs ( $>50 \mathrm{pA})$ resembling the polysynaptic IPSC triggered by an action potential elicited in voltage-clamped cholinergic interneurons. Thus, it is possible that polysynaptic IPSCs are depressed during repetitive firing of cholinergic interneurons. To test this, we evoked a train of action potentials (five at $2 \mathrm{~Hz}$ ) in cholinergic interneurons that produced either feedback $(n=4)$ or feedforward $(n=1)$ IPSCs, and then elicited a single action potential with different intervals (1-10 s) after the train (Fig. 5A). These recordings were done in scopolamine (1 $\mu \mathrm{M})$. The average success rate of a presynaptic action potential to evoke a polysynaptic IPSC was $59 \pm 8 \%$ for the first spike of the train $(n=5)$ (Fig. $5 B, C)$. However, subsequent spikes in the train completely failed to produce polysynaptic IPSCs, suggesting a failure of firing in GABAergic neurons mediating polysynaptic transmission. The success rate gradually recovered over seconds and reached 
$60-90 \%$ of the initial value after $10 \mathrm{~s}$. There was no detectable change in the amplitude of polysynaptic IPSCs during the recovery period, implying that GABAergic synapses on the postsynaptic cholinergic interneurons were not significantly depressed.

We further investigated this short-term depression by applying a train of five intrastriatal stimuli at $2 \mathrm{~Hz}$. These experiments were done in the presence of DNQX (10 $\mu \mathrm{M})$, scopolamine $(1 \mu \mathrm{M})$, and sulpiride $(10 \mu \mathrm{M})$. In four cells tested at threshold intensity $(100-200 \mu \mathrm{A})$, the first stimulus in the train evoked single-peak IPSCs averaging $210 \pm 52 \mathrm{pA}$, with a latency of $13.3 \pm 0.5 \mathrm{~ms}$. The mean success rate for the first stimulus to trigger a polysynaptic IPSC was $64 \pm 9 \%$. The amplitude, latency, and success rate were comparable with the values for the IPSCs produced by an action potential elicited in a single cholinergic interneuron voltage clamped at $-87 \mathrm{mV}$, suggesting that a single cholinergic fiber, likely severed from the tonically firing soma in a slice preparation, is stimulated at threshold intensity. In line with this idea, the second through fifth stimuli in the train were completely ineffective at triggering IPSCs (Fig. 5D), similar to the spike train evoked in a single cholinergic interneuron. However, when higher stimulus intensity $(500-600 \mu \mathrm{A})$ was used to evoke polysynaptic IPSCs with multiple peaks, the fifth stimulus in the $2 \mathrm{~Hz}$ train still produced robust polysynaptic IPSCs: the charge carried by polysynaptic IPSCs evoked by the fifth stimulus was $40 \pm 9 \%$ of the charge of those evoked by the first stimulus $(n=5)$ (Fig. $5 D, E)$. The onset

latency of polysynaptic IPSCs was prolonged from $8.9 \pm 0.5 \mathrm{~ms}$ for the first stimulus to $13.6 \pm 0.7 \mathrm{~ms}$ for the fifth stimulus $(p<$ 0.01 ), suggesting that nAChR-mediated excitation of GABAergic neurons was reduced during the train. Although monosynaptic IPSCs also displayed some depression during the $2 \mathrm{~Hz}$ train, the magnitude of depression was much smaller than that for polysynaptic IPSCs: the amplitude of monosynaptic IPSCs elicited by the fifth stimulus was $77 \pm 7 \%$ of the amplitude of those caused by the first stimulus $(n=4)$.

Together, these results demonstrate that repetitive firing of cholinergic interneurons leads to depression of polysynaptic $\mathrm{GABA}_{\mathrm{A}}$ IPSCs, mostly because of a reduction in cholinergic excitation of GABAergic neurons without much depression of GABAergic synapses onto the postsynaptic cholinergic interneurons. However, simultaneous activation of multiple cholinergic fibers can partially overcome this depression and produce sufficient nAChR-dependent depolarizations in GABAergic neurons, thus reliably triggering polysynaptic IPSCs in cholinergic interneurons.

\section{Polysynaptic inhibition leads to suppression of tonic cholinergic interneuron firing}

Striatal cholinergic interneurons (i.e., TANs) display a pause of tonic firing in response to sensory stimuli during associative learning in vivo (Aosaki et al., 1994b; Apicella, 2007). To examine how polysyn- aptic inhibition affects cholinergic neuron activity, we tested the effect of intrastriatal stimulation on the spontaneous firing of cholinergic interneurons recorded using cell-attached configuration. Firing recordings were done in DNQX $(10 \mu \mathrm{M})$ to prevent timelocked action potentials triggered by monosynaptic EPSPs, and also in eticlopride (100-200 nM) to eliminate the potential contribution of $\mathrm{D}_{2}$ receptor-dependent inhibition of $\mathrm{Na}^{+}$channels in cholinergic interneurons resulting from stimulation of dopaminergic fibers (Maurice et al., 2004). Under these conditions, intrastriatal stimulation $(400-600 \mu \mathrm{A})$ produced a pronounced suppression of tonic firing that started $\sim 20 \mathrm{~ms}$ after the stimulus and lasted for $\sim 250 \mathrm{~ms}$ (Fig. 6). Furthermore, $\mathrm{GABA}_{\mathrm{A}}$ antagonists, GABAzine $(10 \mu \mathrm{M})$ or picrotoxin $(100 \mu \mathrm{M})$, and a general $\mathrm{nAChR}$ antagonist, hexamethonium $(50 \mu \mathrm{M})$, blocked this suppression of tonic firing. None of these drugs significantly changed the baseline firing frequency. These results demonstrate that $\mathrm{nAChR}$-mediated polysynaptic GABAergic inhibition produces a transient suppression of cholinergic interneuron firing.

\section{Discussion}

The main finding of the present study is that striatal cholinergic interneurons communicate with each other via GABAergic neurons. This recurrent polysynaptic connection among cholinergic interneurons is dependent on the activation of $\beta 2$-containing nAChRs. 


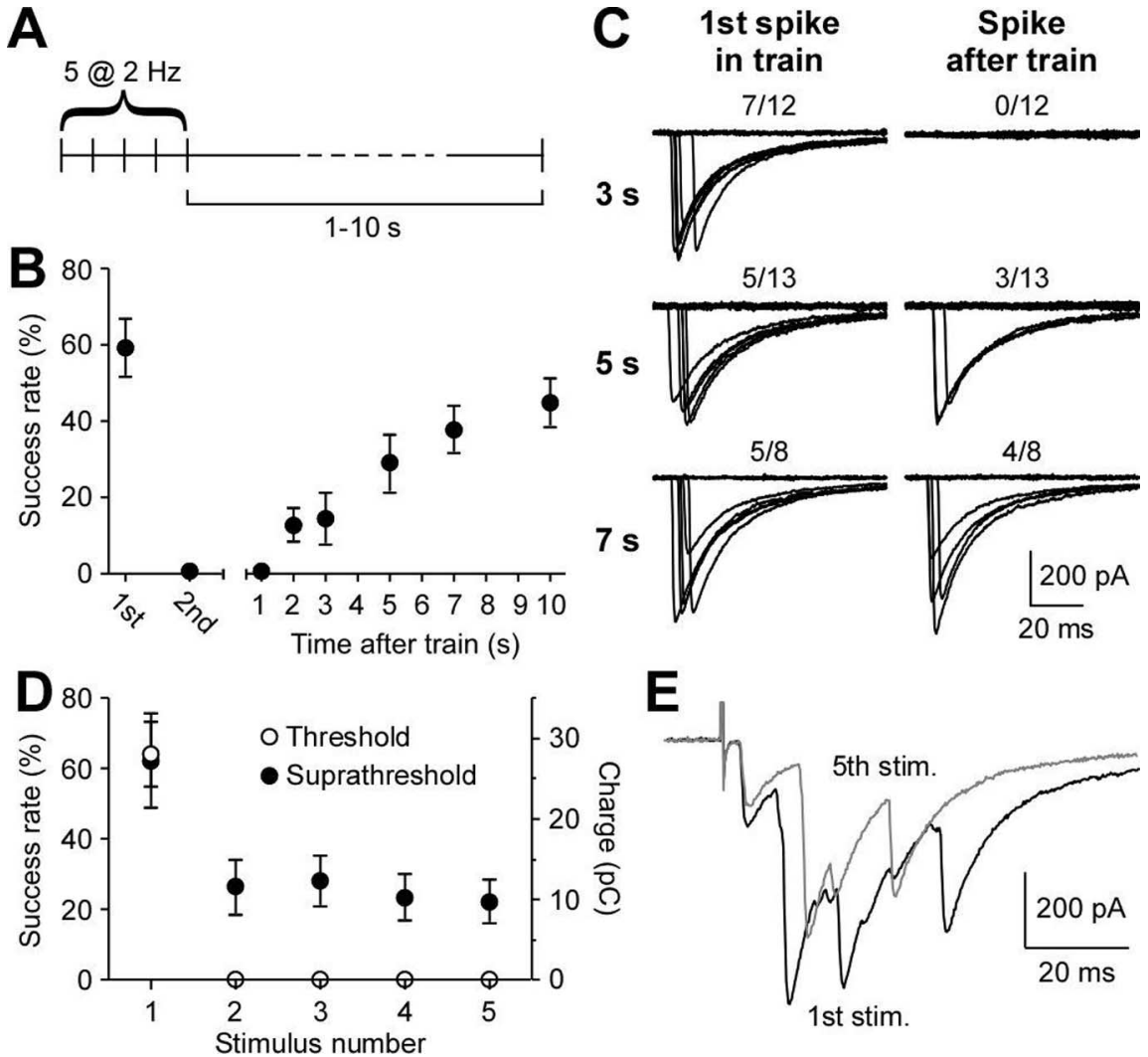

Figure 5. Short-term depression of polysynaptic IPSCs. A, Illustration of the protocol used to evoke a train of action potentials in presynaptic cholinergic interneurons. Depolarizing pulses were applied in a train of five at $2 \mathrm{~Hz}$, followed by a single pulse applied at various intervals after the train. $\boldsymbol{B}$, Summary data from five cells tested with the protocol depicted in $\boldsymbol{A}$. The success rate for a presynaptic action potential to evoke an IPSC is plotted for the first and second spikes in the train and for the single spikes evoked at different intervals (1-10 s) after the train. Data for the third through fifth spikes in the train, which always failed to evoke IPSCS, are not shown for simplicity. $\boldsymbol{C}$, Traces of IPSCs from an experiment using the protocol in $\boldsymbol{A}$. Feedforward PSCS were evoked in this particular experiment. In each row, traces of IPSCS are shown superimposed for the first spike in the train (left column) and for the single spike evoked at the indicated interval after the train (right column). The number of successes out of the number of trials is indicated above each group of traces. $\boldsymbol{D}$, Summary graph illustrating short-term depression of polysynaptic IPSCs evoked by a train of five intrastriatal stimuli at $2 \mathrm{~Hz}$. The success rate for each stimulus to elicit a polysynaptic IPSC is plotted for experiments in which IPSCs were evoked using threshold stimulus intensity $(n=4)$, whereas the charge carried by polysynaptic IPSCs is plotted for experiments using suprathreshold stimulus intensity $(n=5)$. $\boldsymbol{E}$, Representative traces of IPSCs induced by the first and fifth stimuli in the $2 \mathrm{~Hz}$ train using suprathreshold intensity.

Although nAChR-mediated excitation of GABAergic neurons is depressed during repetitive cholinergic interneuron firing, simultaneous activation of many cholinergic fibers by intrastriatal stimulation reliably evokes polysynaptic $\mathrm{GABA}_{\mathrm{A}}$ IPSCs in cholinergic interneurons, resulting in a transient suppression of tonic firing. These findings illustrate a novel microcircuit within the striatum that exerts powerful control over the firing activity of cholinergic interneurons.

\section{The mechanism of polysynaptic transmission}

Polysynaptic GABA IPSCs, described here, have not been observed in previous studies recording synaptic responses of cholinergic interneurons in striatal slices (Bennett and Wilson, 1998; Calabresi et al., 1998; Pisani et al., 2000; Momiyama and Koga, 2001; Centonze et al., 2003; Pakhotin and Bracci, 2007). Although the exact reason for the discrepancy is unclear, it may be caused by the difference in experimental conditions, such as temperature, location, and type of the stimulating electrode, slice plane, and use of MK-801 as an NMDA receptor antagonist, which actually blocks nAChRs, as well (Amador and Dani, 1991; Yamakura et al., 2000). Of particular interest is the previous re- port demonstrating that stimulation of the subcortical white matter containing both corticostriatal and thalamostriatal glutamatergic fibers results in polysynaptic GABAergic inhibition through activation of AMPA receptors (Suzuki et al., 2001). This polysynaptic GABAergic inhibition was observed in only $\sim 10 \%$ of the recorded cholinergic interneurons, whereas polysynaptic IPSCs were invariably evoked in our case using intrastriatal stimulation. It is possible that our results have characterized part of the pathway underlying the polysynaptic IPSCs described in this previous study. In this scenario, coincident glutamatergic excitation presumably evokes synchronous firing of a population of cholinergic interneurons via AMPA receptors, leading to $\mathrm{nAChR}$ dependent excitation of GABAergic neurons and subsequent inhibition of cholinergic interneurons.

Dopamine receptor antagonists or dopamine depletion did not affect polysynaptic GABAergic inhibition. This result rules out the scheme in which stimulation of cholinergic fibers indirectly excites striatal GABAergic neurons via phasic dopamine release triggered by activation of $\beta 2$ containing nAChRs on dopaminergic terminals (Zhou et al., 2001; Rice and Cragg, 2004; Zhang and Sulzer, 2004). Therefore, it is most likely that cholinergic fibers make direct synaptic contacts onto GABAergic neurons that in turn innervate cholinergic interneurons, as illustrated in Figure 4C. Dopamine has been shown to play an important role in the pause response of tonically active cholinergic interneurons in behaving animals (Aosaki et al., 1994a; Watanabe and Kimura, 1998). However, phasic dopamine release in the striatum may not directly trigger the pause, because it can be observed in the absence of phasic dopamine response under certain conditions during sensorimotor conditioning (Morris et al., 2002 , 2004). Moreover, systemic administration of a general dopamine receptor agonist, apomorphine, can restore the pause response suppressed by infusion of dopaminergic neurotoxin into the striatum (Aosaki et al., 1994a), suggesting a permissive role of tonic dopamine in enabling pause generation.

Evoking an action potential in a single cholinergic interneuron produced polysynaptic IPSCs in itself and nearby cholinergic interneurons. It has been reported recently that firing of a single cholinergic interneuron leads to suppression of glutamatergic and GABAergic transmission onto neighboring spiny projection neurons and cholinergic interneurons via activation of muscarinic ACh receptors (Narushima et al., 2007; Pakhotin and Bracci, 2007). Our results demonstrate that activation of a single cholinergic interneuron can also drive excitation of surrounding GABAergic neurons through activation of nAChRs. The latency of polysynaptic IPSCs triggered by single cholinergic neuron activation, as well as those evoked by threshold intrastriatal stimulation, was fairly long $(\sim 14-16 \mathrm{~ms})$, which presumably reflects 
the time for relatively small nicotinic EPSPs to elicit a spike in GABAergic neurons. Although polysynaptic connectivity between single cholinergic interneurons was sparse $(\sim 3 \%)$, stimulation of intrastriatal cholinergic fibers was capable of eliciting polysynaptic IPSCs in all cholinergic interneurons tested in this study. Hence, each cholinergic interneuron invariably receives input from those GABAergic neurons that are activated by local cholinergic input. In other words, each cholinergic interneuron can be polysynaptically influenced by the activity of other cholinergic interneurons, likely reflecting extensive axonal arborizations of GABAergic neurons as well as cholinergic interneurons.

Our results further showed that repetitive firing of cholinergic interneurons at 2 $\mathrm{Hz}$ induces depression of nAChRmediated transmission onto GABAergic neurons. This ensures that polysynaptic inhibition of cholinergic interneurons is not constantly triggered by the spontaneous activity of individual cholinergic interneurons. Therefore, synchronous activation of multiple cholinergic interneurons is necessary to overcome this depression and mediate efficient inhibition of cholinergic neuron excitability. It remains to be determined whether this short-term depression is caused by presynaptic depression of ACh release, postsynaptic desensitization of nAChRs, or both. Activity dependence of cholinergic transmission in the CNS is not well known, mainly because of the difficulty in evoking cholinergic synaptic responses in brain slices (Dani and Bertrand, 2007). $\beta 2$-Containing nAChRs are readily desensitized by a small but sustained elevation of ACh levels (Giniatullin et al., 2005). However, removal of ACh by acetylcholinesterase at synapses is thought to be very rapid ( $\sim 1 \mathrm{~ms}$ ), thereby precluding significant $\mathrm{nAChR}$ desensitization by synaptically released ACh (Dani et al., 2001). In line with this, $\beta 2$-containing nAChRs on dopaminergic terminals remain in a non-desensitized, functional state in the striatum despite the constant release of ACh resulting from the tonic activity of cholinergic interneurons (Zhou et al., 2001). Thus, presynaptic depression may play a more dominant role in the short-term depression of cholinergic transmission onto GABAergic neurons. Indeed, repetitive stimulation at $2-20 \mathrm{~Hz}$ has been shown to cause shortterm depression of nicotinic EPSPs via a presynaptic mechanism in cervical sympathetic ganglion neurons (Birks and Isacoff, 1988).

The present study strongly suggests the presence of GABAergic neurons expressing $\beta 2$-containing $\mathrm{nAChRs}$ in the striatum. In addition to GABAergic projection neurons, a small percentage $(2-3 \%)$ of the striatal neuronal population comprises different
A

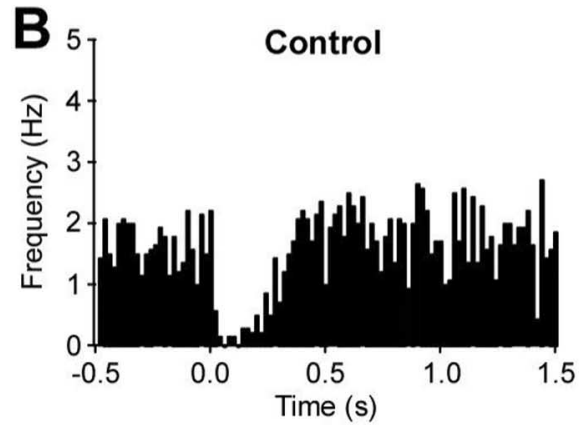

C
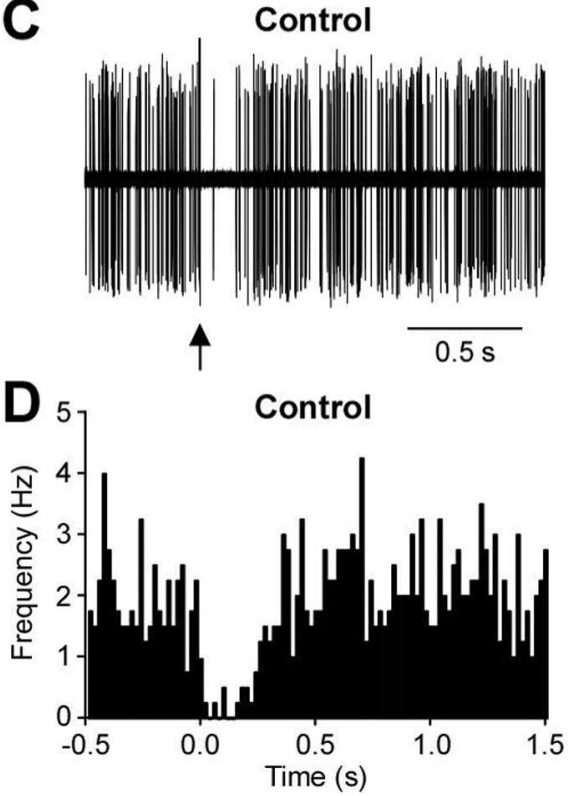

Control

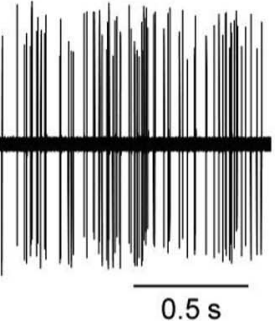

Contro
Figure 6. Polysynaptic GABA IPSCs cause transient suppression of spontaneous cholinergic interneuron firing. $A$, Representative traces of the firing activity of a cholinergic interneuron in control and in GABAzine $(10 \mu \mathrm{M})$. Fifty traces are overlaid in each condition. Intrastriatal stimulation was applied at the time indicated by the arrow. The baseline firing frequency was $1.1 \mathrm{~Hz}$ and $1.2 \mathrm{~Hz}$ in control and in GABAzine, respectively. $\boldsymbol{B}$, Peristimulus time histograms (bin width, $20 \mathrm{~ms}$ ) of cholinergic interneuron firing in control and in GABAzine $(10 \mu \mathrm{m})$ or picrotoxin $(100 \mu \mathrm{m})$. Intrastriatal stimulus was applied at time 0 . The data were averaged from seven cells. The duration of the firing suppression in control was $260 \mathrm{~ms}$ in these seven cells. C, Overlay of 50 traces of the firing of a cholinergic interneuron in control and in hexamethonium $(50 \mu \mathrm{m})$. The baseline firing frequency was $3.0 \mathrm{~Hz}$ and $3.3 \mathrm{~Hz}$ in control and in hexamethonium, respectively. $\boldsymbol{D}$, Averaged histograms in control and in hexamethonium ( $50 \mu \mathrm{M})$ from four cells. The duration of the firing suppression was $220 \mathrm{~ms}$ in these four cells.

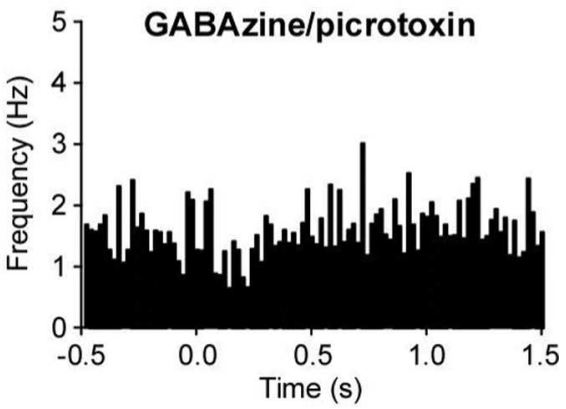

Hexamethonium

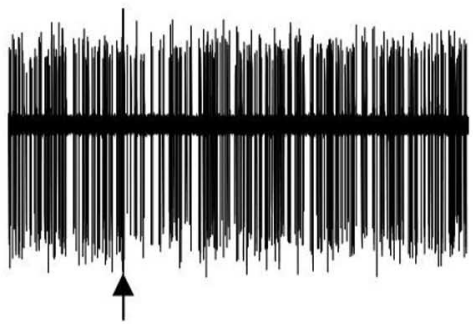

Hexamethonium

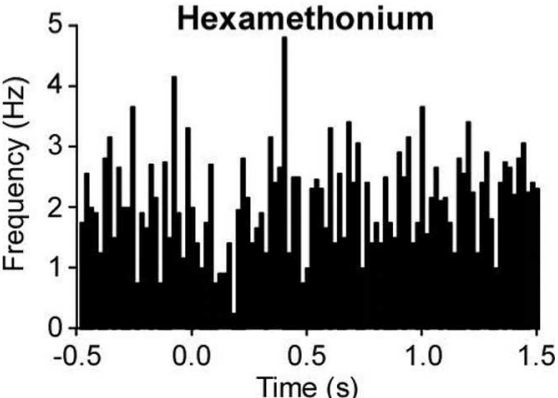

types of GABAergic interneurons (Tepper and Bolam, 2004). Detailed histochemical evidence indicates that $\mathrm{nAChR} \beta 2$-subunit immunoreactivity cannot be detected in spiny projection neurons (Hill et al., 1993; Jones et al., 2001). nAChR-mediated depolarization has been recently shown in fast-spiking (FS) interneurons; however, pharmacological examination has ruled out the involvement of $\beta 2$-containing nAChRs (Koós and Tepper, 2002). Furthermore, it has been reported that synaptic connectivity between cholinergic and FS interneurons is unidirectional, in that cholinergic terminals make synaptic contacts onto 
parvalbumin-positive FS interneurons but not vice versa (Chang and Kita, 1992). However, reciprocal synaptic contacts between cholinergic interneurons and neuropeptide Y-containing neurons, which corresponds to persistent and low-threshold spike (PLTS) interneurons, has been detected (Vuillet et al., 1992). It should also be noted that PLTS interneurons have depolarized resting membrane potential $(-50$ to $-60 \mathrm{mV}$ ) close to action potential threshold and very-high-input resistance (>500 M $\Omega$ ) (Kawaguchi, 1993; Tepper and Bolam, 2004). These intrinsic membrane properties, together with their elongated axonal arbors (up to $1 \mathrm{~mm}$ ) (Kawaguchi, 1993; Kubota and Kawaguchi, 2000), would make PLTS interneurons suited for transforming small excitatory cholinergic input into extensive inhibition of downstream neurons.

\section{Functional significance of recurrent inhibition among cholinergic interneurons}

It is well established that the pause response of cholinergic interneurons, or TANs, to sensory cues develops during reward-based conditioning (Aosaki et al., 1994b; Morris et al., 2004), and the population response of these neurons has been linked to the behavioral output (Blazquez et al., 2002; Apicella, 2007). Previous studies in vivo have indicated the important roles of glutamatergic and dopaminergic inputs in the generation of the pause (Aosaki et al., 1994a; Watanabe and Kimura, 1998; Matsumoto et al., 2001; Reynolds et al., 2004). Accordingly, a number of studies in vitro have addressed the potential mechanisms underlying the pause based on the premise that it results from the interplay among glutamatergic and dopaminergic inputs, local GABAergic transmission, and intrinsic cholinergic neuron properties (Yan and Surmeier, 1997; Bennett and Wilson, 1998; Suzuki et al., 2001; Maurice et al., 2004; Wilson, 2005; Deng et al., 2007). However, a pause mimicking the in vivo pause has not been readily induced by synaptic stimulation in brain slices. In this study, under AMPA receptor blockade to prevent time-locked action potentials and subsequent prolonged afterhyperpolarizations (Bennett et al., 2000), intrastriatal stimulation of cholinergic fibers reliably produced a transient suppression of tonic cholinergic interneuron firing for $\sim 250 \mathrm{~ms}$, which is comparable with the duration of the pause response in behaving animals (Aosaki et al., 1995; Morris et al., 2004). The pause response in vivo is frequently preceded by a small, brief $(<50 \mathrm{~ms})$ increase in firing frequency (Apicella, 2007), which represents roughly synchronized firing of a population of cholinergic interneurons within a short time window. Successive, small nicotinic EPSPs during this time window may well summate to produce sizable depolarizations in GABAergic interneurons, thereby driving their firing. Therefore, the polysynaptic inhibitory mechanism described in this study may contribute, at least partially, to the generation of pause by transforming activation of a population of cholinergic interneurons into recurrent inhibition of a larger population of these neurons.

\section{References}

Amador M, Dani JA (1991) MK-801 inhibition of nicotinic acetylcholine receptor channels. Synapse 7:207-215.

Aosaki T, Graybiel AM, Kimura M (1994a) Effect of the nigrostriatal dopamine system on acquired neural responses in the striatum of behaving monkeys. Science 265:412-415.

Aosaki T, Tsubokawa H, Ishida A, Watanabe K, Graybiel AM, Kimura M (1994b) Responses of tonically active neurons in the primate's striatum undergo systematic changes during behavioral sensorimotor conditioning. J Neurosci 14:3969-3984.

Aosaki T, Kimura M, Graybiel AM (1995) Temporal and spatial character- istics of tonically active neurons of the primate's striatum. J Neurophysiol 73:1234-1252.

Apicella P (2007) Leading tonically active neurons of the striatum from reward detection to context recognition. Trends Neurosci 30:299-306.

Bennett BD, Wilson CJ (1998) Synaptic regulation of action potential timing in neostriatal cholinergic interneurons. J Neurosci 18:8539-8549.

Bennett BD, Callaway JC, Wilson CJ (2000) Intrinsic membrane properties underlying spontaneous tonic firing in neostriatal cholinergic interneurons. J Neurosci 20:8493-8503.

Birks RI, Isacoff EY (1988) Burst-patterned stimulation promotes nicotinic transmission in isolated perfused rat sympathetic ganglia. J Physiol 402:515-532.

Blazquez PM, Fujii N, Kojima J, Graybiel AM (2002) A network representation of response probability in the striatum. Neuron 33:973-982.

Calabresi P, Centonze D, Pisani A, Sancesario G, North RA, Bernardi G (1998) Muscarinic IPSPs in rat striatal cholinergic interneurones. J Physiol 510:421-427.

Calabresi P, Centonze D, Gubellini P, Pisani A, Bernardi G (2000) Acetylcholine-mediated modulation of striatal function. Trends Neurosci 23:120-126.

Centonze D, Grande C, Usiello A, Gubellini P, Erbs E, Martin AB, Pisani A, Tognazzi N, Bernardi G, Moratalla R, Borrelli E, Calabresi P (2003) Receptor subtypes involved in the presynaptic and postsynaptic actions of dopamine on striatal interneurons. J Neurosci 23:6245-6254.

Chang HT, Kita H (1992) Interneurons in the rat striatum: relationships between parvalbumin neurons and cholinergic neurons. Brain Res 574:307-311.

Cruikshank SJ, Rose HJ, Metherate R (2002) Auditory thalamocortical synaptic transmission in vitro. J Neurophysiol 87:361-384.

Cui G, Bernier BE, Harnett MT, Morikawa H (2007) Differential regulation of action potential- and metabotropic glutamate receptor-induced $\mathrm{Ca} 2+$ signals by inositol 1,4,5-trisphosphate in dopaminergic neurons. J Neurosci $27: 4776-4785$.

Dani JA, Bertrand D (2007) Nicotinic acetylcholine receptors and nicotinic cholinergic mechanisms of the central nervous system. Annu Rev Pharmacol Toxicol 47:699-729.

Dani JA, Ji D, Zhou FM (2001) Synaptic plasticity and nicotine addiction. Neuron 31:349-352.

Deng P, Zhang Y, Xu ZC (2007) Involvement of I(h) in dopamine modulation of tonic firing in striatal cholinergic interneurons. J Neurosci $27: 3148-3156$

Giniatullin R, Nistri A, Yakel JL (2005) Desensitization of nicotinic ACh receptors: shaping cholinergic signaling. Trends Neurosci 28:371-378.

Graybiel AM, Aosaki T, Flaherty AW, Kimura M (1994) The basal ganglia and adaptive motor control. Science 265:1826-1831.

Hill JA Jr, Zoli M, Bourgeois JP, Changeux JP (1993) Immunocytochemical localization of a neuronal nicotinic receptor: the beta 2-subunit. J Neurosci 13:1551-1568.

Jones IW, Bolam JP, Wonnacott S (2001) Presynaptic localisation of the nicotinic acetylcholine receptor beta2 subunit immunoreactivity in rat nigrostriatal dopaminergic neurones. J Comp Neurol 439:235-247.

Kawaguchi Y (1993) Physiological, morphological, and histochemical characterization of three classes of interneurons in rat neostriatum. J Neurosci 13:4908-4923.

Kita H (2007) Globus pallidus external segment. Prog Brain Res 160:111-133.

Kitabatake Y, Hikida T, Watanabe D, Pastan I, Nakanishi S (2003) Impairment of reward-related learning by cholinergic cell ablation in the striatum. Proc Natl Acad Sci U S A 100:7965-7970.

Koós T, Tepper JM (2002) Dual cholinergic control of fast-spiking interneurons in the neostriatum. J Neurosci 22:529-535.

Kubota Y, Kawaguchi Y (2000) Dependence of GABAergic synaptic areas on the interneuron type and target size. J Neurosci 20:375-386.

Lapper SR, Bolam JP (1992) Input from the frontal cortex and the parafascicular nucleus to cholinergic interneurons in the dorsal striatum of the rat. Neuroscience 51:533-545.

Liao X, Walters ET (2002) The use of elevated divalent cation solutions to isolate monosynaptic components of sensorimotor connections in Aplysia. J Neurosci Methods 120:45-54.

Mansvelder HD, Keath JR, McGehee DS (2002) Synaptic mechanisms underlie nicotine-induced excitability of brain reward areas. Neuron 33:905-919. 
Matsumoto N, Minamimoto T, Graybiel AM, Kimura M (2001) Neurons in the thalamic CM-Pf complex supply striatal neurons with information about behaviorally significant sensory events. J Neurophysiol 85:960-976.

Maurice N, Mercer J, Chan CS, Hernandez-Lopez S, Held J, Tkatch T, Surmeier DJ (2004) D2 dopamine receptor-mediated modulation of voltage-dependent $\mathrm{Na}+$ channels reduces autonomous activity in striatal cholinergic interneurons. J Neurosci 24:10289-10301.

Momiyama T, Koga E (2001) Dopamine D(2)-like receptors selectively block $\mathrm{N}$-type $\mathrm{Ca}(2+)$ channels to reduce GABA release onto rat striatal cholinergic interneurones. J Physiol 533:479-492.

Morris G, Raz A, Arkadir D, Bergman H (2002) Striatal TANs do not report prediction error. In: The basal ganglia VII. Advances in behavioral biology, Vol 52 (Nicholson LFB, Faull RLM, eds), pp 173-179. New York: Springer.

Morris G, Arkadir D, Nevet A, Vaadia E, Bergman H (2004) Coincident but distinct messages of midbrain dopamine and striatal tonically active neurons. Neuron 43:133-143.

Narushima M, Uchigashima M, Fukaya M, Matsui M, Manabe T, Hashimoto K, Watanabe M, Kano M (2007) Tonic enhancement of endocannabinoid-mediated retrograde suppression of inhibition by cholinergic interneuron activity in the striatum. J Neurosci 27:496-506.

Pakhotin P, Bracci E (2007) Cholinergic interneurons control the excitatory input to the striatum. J Neurosci 27:391-400.

Pisani A, Bonsi P, Centonze D, Calabresi P, Bernardi G (2000) Activation of D2-like dopamine receptors reduces synaptic inputs to striatal cholinergic interneurons. J Neurosci 20:RC69(1-6).

Reynolds JN, Wickens JR (2004) The corticostriatal input to giant aspiny interneurons in the rat: a candidate pathway for synchronising the response to reward-related cues. Brain Res 1011:115-128.

Reynolds JN, Hyland BI, Wickens JR (2004) Modulation of an afterhyperpolarization by the substantia nigra induces pauses in the tonic firing of striatal cholinergic interneurons. J Neurosci 24:9870-9877.

Rice ME, Cragg SJ (2004) Nicotine amplifies reward-related dopamine signals in striatum. Nat Neurosci 7:583-584.

Suzuki T, Miura M, Nishimura K, Aosaki T (2001) Dopamine-dependent synaptic plasticity in the striatal cholinergic interneurons. J Neurosci 21:6492-6501.
Tepper JM, Bolam JP (2004) Functional diversity and specificity of neostriatal interneurons. Curr Opin Neurobiol 14:685-692.

Tepper JM, Koós T, Wilson CJ (2004) GABAergic microcircuits in the neostriatum. Trends Neurosci 27:662-669.

Vuillet J, Dimova R, Nieoullon A, Kerkerian-Le Goff L (1992) Ultrastructural relationships between choline acetyltransferase- and neuropeptide $y$-containing neurons in the rat striatum. Neuroscience 46:351-360.

Wang Z, Kai L, Day M, Ronesi J, Yin HH, Ding J, Tkatch T, Lovinger DM Surmeier DJ (2006) Dopaminergic control of corticostriatal long-term synaptic depression in medium spiny neurons is mediated by cholinergic interneurons. Neuron 50:443-452.

Watanabe K, Kimura M (1998) Dopamine receptor-mediated mechanisms involved in the expression of learned activity of primate striatal neurons. J Neurophysiol 79:2568-2580.

Wilson CJ (2005) The mechanism of intrinsic amplification of hyperpolarizations and spontaneous bursting in striatal cholinergic interneurons. Neuron 45:575-585.

Wilson CJ, Chang HT, Kitai ST (1990) Firing patterns and synaptic potentials of identified giant aspiny interneurons in the rat neostriatum. J Neurosci 10:508-519.

Yamakura T, Chavez-Noriega LE, Harris RA (2000) Subunit-dependent inhibition of human neuronal nicotinic acetylcholine receptors and other ligand-gated ion channels by dissociative anesthetics ketamine and dizocilpine. Anesthesiology 92:1144-1153.

Yan Z, Surmeier DJ (1997) D5 dopamine receptors enhance Zn2+sensitive GABA(A) currents in striatal cholinergic interneurons through a PKA/PP1 cascade. Neuron 19:1115-1126.

Zhang H, Sulzer D (2004) Frequency-dependent modulation of dopamine release by nicotine. Nat Neurosci 7:581-582.

Zhou FM, Liang Y, Dani JA (2001) Endogenous nicotinic cholinergic activity regulates dopamine release in the striatum. Nat Neurosci 4:1224-1229.

Zhou FM, Wilson CJ, Dani JA (2002) Cholinergic interneuron characteristics and nicotinic properties in the striatum. J Neurobiol 53:590-605.

Zhou FM, Wilson C, Dani JA (2003) Muscarinic and nicotinic cholinergic mechanisms in the mesostriatal dopamine systems. Neuroscientist 9:2336. 